\title{
Rapidly reconfigurable slow-light system based on off-resonant Raman absorption
}

\author{
Praveen K. Vudyasetu, ${ }^{1, *}$ Ryan M. Camacho, ${ }^{1,2}$ and John C. Howell ${ }^{1}$ \\ ${ }^{1}$ Department of Physics and Astronomy, University of Rochester, Rochester, New York 14627, USA \\ ${ }^{2}$ Thomas J. Watson, Sr., Laboratory of Applied Physics, California Institute of Technology, Pasadena, California 91125, USA
}

(Received 4 August 2010; published 9 November 2010)

\begin{abstract}
We present a slow-light system based on dual Raman absorption resonances in warm rubidium vapor. Each Raman absorption resonance is produced by a control beam in an off-resonant $\Lambda$ system. This system combines all optical control of the Raman absorption and the low-dispersion broadening properties of the double Lorentzian absorption slow light. The bandwidth, group delay, and central frequency of the slow-light system can all be tuned dynamically by changing the properties of the control beam. We demonstrate multiple pulse delays with low distortion and show that such a system has fast switching dynamics and thus fast reconfiguration rates.
\end{abstract}

DOI: 10.1103/PhysRevA.82.053807

PACS number(s): 42.65.-k

\section{INTRODUCTION}

The ability to control the group velocity of a light pulse is central to the practical realizations of all optical communication systems [1]. An ideal slow-light system must have a large delay-bandwidth product with fast tunability, large bandwidth, low distortion, and low absorptive losses [2-4]. After an initial demonstration of ultraslow group velocities of light pulses by Hau et al. [5], several groups have demonstrated slow light in a wide variety of media, such as atomic vapors [6-8], solid materials [9-11], optical fibers [12], liquid crystals [13], and photonic crystals [14]. Slow light has applications in many diverse fields, such as optical communications [1], interferometry [15,16], and sensing [17].

An optical signal pulse with central frequency $\omega_{s}$ propagates slowly in a medium of refractive index $n(\omega)$ if its group index defined as $n_{g}=\left.\omega_{s} \frac{\partial n(\omega)}{\partial \omega}\right|_{\omega=\omega_{s}}$ is very large. One can obtain very large group indices in the vicinity of a sharp transmission at the signal frequency. Such sharp transmissions can be achieved by using a variety of nonlinear effects in atomic media [2] or by fabricating specially designed photonic structures [14]. In this paper, we deal with the former case, that is, obtaining slow light by tailoring the refractive index of the medium.

Camacho et al. [8] have shown that one can achieve multiple pulse delays by operating between two Lorentzian absorption resonances. Such a double-Lorentzian system is shown to dramatically reduce absorptive broadening and to cancel the dispersive broadening and, thus, is advantageous over a singleLorentzian system, such as an electromagnetically induced transparency (EIT) system. Slow light is primarily limited by absorptive broadening in addition to absorptive losses in such a double-Lorentzian system. The depth and linewidth of each of the resonances along with the separation between two resonances determine the system bandwidth, the group delay, and the absorptive losses. The double-absorption slow light was also demonstrated in fibers using anti-Stokes absorption resonances by Zhu and Gauthier [18].

For the purposes of optical communication systems and other applications, rapid tunability of the group velocity of a light pulse is advantageous. For example, one could use such

*praveen@pas.rochester.edu a system to synchronize a pulse train by tailoring the group velocity of each pulse in the pulse train. Such an application demands the group velocity switching times to be less than the signal pulse width. We can also use it to get single photons on demand, by slowing down the single photons and turning off the delay at the appropriate time. For applications such as slow-light enhanced Fourier transform interferometry [15], the time required to determine the spectrum of an unknown signal is directly proportional to the group velocity switching time.

In this paper, we describe a fast, optically tunable doubleabsorption slow-light system where the absorption resonance for the probe is artificially created by a coupling beam in a three-level off-resonant $\Lambda$ system. We show that the properties of each of the absorption lines can be changed optically by tuning the properties of the coupling beams which create them. The resonant $\Lambda$ system has been studied extensively in the context of EIT [19]. The Raman absorption line produced by an off-resonant $\Lambda$ system has been studied and used to produce fast light $[20,21]$. Such a process has also been used by Reim et al. to store very weak signal pulses [22]. Proite et al. created a gain line and an absorption line using two coupling lasers in order to obtain an enhanced refractive index [23]. Here we propose to use two absorption lines in order to realize a double-Lorentzian slow-light system. We also study the transient response to the switching of the coupling beam properties and show that one can dynamically and rapidly tune the group delays.

\section{THEORY}

Consider the three-level $\Lambda$ system shown in Fig. 1(b). Levels 1 and 2 are connected by the probe field $\frac{\Omega_{p}}{2} e^{-i\left(\omega_{p} t-k z\right)}+$ c.c. The control field connecting states 2 and 3 can be written as $\frac{\Omega_{c}}{2} e^{-i\left(\omega_{c} t-k z\right)}+$ c.c. The detunings are defined as $\Delta=\Delta_{p}=$ $\left(\omega_{2}-\omega_{1}\right)-\omega_{p}, \Delta_{c}=\left(\omega_{2}-\omega_{3}\right)-\omega_{c}$, and $\delta=\Delta_{p}-\Delta_{c}$.

The susceptibility at probe frequency can be obtained as

$$
\chi\left(\Delta, \delta, \Omega_{c}\right)=\beta \frac{\delta-i \gamma}{(\delta-i \gamma)(\Delta-i \Gamma / 2)-\left|\Omega_{c}\right|^{2} / 4},
$$

where $\Gamma$ and $\gamma$ are the excited-state decay rate and ground-state decoherence rate, respectively, and $\beta=N \mu^{2} / \hbar \epsilon_{0}$, where $N$ is the number density and $\mu$ is the transition dipole moment.

Now consider the case where the probe has to propagate through two identically prepared vapor cells. The effective 
(a)

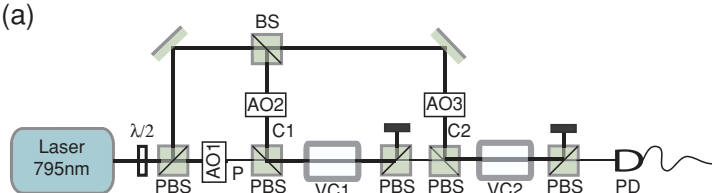

(b)

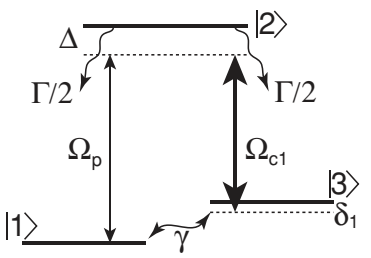

VC1 (c)

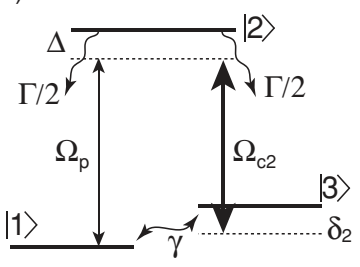

VC2

FIG. 1. (Color online) (a) The outline of the setup used in the experiments, and the energy level diagrams for vapor cells (b) VC1 and (c) VC2.

susceptibility at the probe frequency is given by the sum of susceptibilities in each of the cells. The control beams in the two cells only differ by frequency $2 \delta_{c}$. The effective susceptibility is given by $\chi^{\text {eff }}\left(\Delta, \delta, \Omega_{c}, \delta_{c}\right)=\chi\left(\Delta, \delta-\delta_{c}, \Omega_{c}\right)+\chi(\Delta, \delta+$ $\left.\delta_{c}, \Omega_{c}\right)$, where $\chi\left(\Delta, \delta, \Omega_{c}\right)$ is given by Eq. (1). Expanding the refractive index around $\delta=\left|\Omega_{c}\right|^{2} \Delta /\left(4 \Delta^{2}+\Gamma^{2}\right)$, we obtain the following expressions for real and imaginary parts of the refractive index:

$$
\begin{aligned}
n^{\prime}= & n_{0}^{\prime}-\frac{C_{2}}{\delta_{c}}\left[\frac{\delta^{\prime}}{\delta_{c}}+\left(\frac{\delta^{\prime}}{\delta_{c}}\right)^{3}+\cdots\right] \\
& -\frac{C_{1} \gamma^{\prime}}{\delta_{c}^{2}}\left[1+3\left(\frac{\delta^{\prime}}{\delta_{c}}\right)^{2}+\cdots\right], \\
n^{\prime \prime}= & n_{0}^{\prime \prime}-\frac{C_{1}}{\delta_{c}}\left[\frac{\delta^{\prime}}{\delta_{c}}+\left(\frac{\delta^{\prime}}{\delta_{c}}\right)^{3}+\cdots\right] \\
& +\frac{C_{2} \gamma^{\prime}}{\delta_{c}^{2}}\left[1+3\left(\frac{\delta^{\prime}}{\delta_{c}}\right)^{2}+\cdots\right] .
\end{aligned}
$$

The effective detuning and the effective linewidth are given by $\delta^{\prime}=\delta-\delta_{0}$ and $\gamma^{\prime}=\gamma+\gamma_{0}$, respectively, where $\delta_{0}=$ $\left|\Omega_{c}\right|^{2} \Delta /\left(4 \Delta^{2}+\Gamma^{2}\right)$ and $\gamma_{0}=\left|\Omega_{c}\right|^{2} \Gamma /\left(8 \Delta^{2}+2 \Gamma^{2}\right)$. The constants are $C_{1}=\beta \frac{\left|\Omega_{c}\right|^{2}}{4} \frac{\Delta \Gamma}{\left(\Delta^{2}+\Gamma^{2} / 4\right)^{2}}$ and $C_{2}=\beta \frac{\left|\Omega_{c}\right|^{2}}{4} \frac{\Delta^{2}-\Gamma^{2} / 4}{\left(\Delta^{2}+\Gamma^{2} / 4\right)^{2}}$. For the off-resonant case where $\Delta \gg \Gamma, C_{1}$ approaches zero faster than $C_{2}$. For this case, we can approximate the off-resonant line shape to a Lorentzian and thus we can obtain a double-Lorentzian system. Following the approach of Camacho et al. [8], we can write the dispersive and absorptive broadening for a pulse with Gaussian envelope $\exp \left[-t^{2} /\left(2 T_{0}^{2}\right)\right]$ after propagation through the medium of length $L$ as

$$
\begin{gathered}
T_{a}^{2}=T_{0}^{2}+\frac{12 \omega_{p} L C_{2} \gamma^{\prime}}{c \delta_{c}^{4}}=T_{0}^{2}+\frac{12 \tau_{d} \gamma^{\prime}}{\delta_{c}^{2}}, \\
T_{d}^{2}=T_{0}^{2}+\left(\frac{3 \omega_{p} C_{2} L}{\delta_{c}^{4} c T_{0}^{2}}\right)^{2}=T_{0}^{2}+\left(\frac{3 \tau_{d}}{\delta_{c}^{2} T_{0}^{2}}\right)^{2} .
\end{gathered}
$$

The relevant slow-light parameters for a propagation distance $L$, the group delay $\tau_{d}$, and absorption $\alpha L$ are

given as

$$
\begin{gathered}
\tau_{d}=\frac{\beta \omega_{p} L}{c} \frac{\left|\Omega_{c}\right|^{2}}{4 \Delta^{2}} \frac{1}{\delta_{c}^{2}}, \\
\alpha L=\frac{\beta \omega_{p} L}{c}\left(\frac{\left|\Omega_{c}\right|^{2}}{4 \Delta^{2}}\right)^{2} \frac{\Gamma}{2 \delta_{c}^{2}} .
\end{gathered}
$$

We define the bandwidth $B$ of the system as the bandwidth of the pulse for which the absorptive broadening is equal to 2. Thus, the bandwidth of the system is given as $B=\delta_{c} / 2$. Also, from the preceding equations we note that $\tau_{d}=\alpha L / \gamma_{0}$ and, hence, the time delay for one absorption length is simply the inverse of the power-broadened linewidth of the Raman absorption resonance. The delay-bandwidth product for one absorption length is thus given by

$$
\tau_{d} \times B=\frac{\delta_{c}}{2 \gamma_{0}} .
$$

The bandwidth of the system can be tuned simply by changing the frequency difference between two control beams and the delay-bandwidth product can be changed by changing the linewidth $\gamma_{0}$ of the Raman resonance; $\gamma_{0}$ can be tailored by changing the single-photon detuning $\Delta$ or by changing the power of the control beams. Thus, we obtain all optical control over the bandwidth and the delay-bandwidth product.

In order to study the time response of the system, we need to solve the dynamic equations for atomic coherence. In the weak perturbation regime for the probe, we write the following equation for the ground-state coherence $\rho_{21}$ :

$$
\begin{gathered}
\ddot{\rho_{21}}+i(\Delta+\delta-i \Gamma / 2-i \gamma) \rho_{21}-[(\Delta-i \Gamma / 2)(\delta-i \gamma) \\
\left.-\left|\Omega_{c}\right|^{2} / 4\right] \rho_{21}+\frac{\Omega_{p}}{2}(\delta-i \gamma)=0 .
\end{gathered}
$$

We first solve the preceding equation for the case where the control beam is turned on at time $t=0$. The solution is of the form

$$
\begin{aligned}
\rho_{21}(t)= & {\left[\frac{\lambda_{-}}{\lambda_{+}-\lambda_{-}} e^{\lambda_{+} t}-\frac{\lambda_{+}}{\lambda_{+}-\lambda_{-}} e^{\lambda_{-} t}\right] } \\
& \times\left(\rho_{21}^{(s s)}-\rho_{21}^{(0)}\right)+\rho_{21}^{(s s)},
\end{aligned}
$$

where $\rho_{21}^{(s s)}$ is the steady-state coherence and $\rho_{21}^{(0)}$ is the steadystate coherence without the control beam on. The constants $\lambda_{+}$ and $\lambda_{-}$for large $\Delta$ are given by

$$
\begin{gathered}
\lambda_{+}=i \frac{\left|\Omega_{c}\right|^{2}}{4 \Delta}-\frac{\left|\Omega_{c}\right|^{2} \Gamma}{8 \Delta^{2}} \\
\lambda_{-}=-i\left(\Delta+\frac{\left|\Omega_{c}\right|^{2}}{4 \Delta}\right)-\frac{\Gamma}{2}\left(1-\frac{\left|\Omega_{c}\right|^{2}}{4 \Delta^{2}}\right) .
\end{gathered}
$$

For $\frac{\left|\Omega_{c}\right|^{2}}{4 \Delta^{2}} \ll 1$, we can see that $\exp \left(\lambda_{-} t\right)$ is rapidly decaying compared to $\exp \left(\lambda_{+} t\right)$. Hence, the characteristic transient time for turning on the slow-light medium is given by reciprocal of real part of $\lambda_{+}$:

$$
\tau^{\text {on }}=\frac{8 \Delta^{2}}{\left|\Omega_{c}\right|^{2} \Gamma}=1 / \gamma_{0} .
$$

In order to calculate the turn-off time, we consider the case where the coherence is $\rho_{21}^{(s s)}$ for $t<0$ and the pump is turned 
off at $t=0$. The time evolution of the coherence for $t>0$ is given by

$$
\rho_{21}(t)=\left(\rho_{21}^{(s s)}-\rho_{21}^{(0)}\right) e^{-(i \Delta+\Gamma / 2) t}+\rho_{21}^{(0)} .
$$

The characteristic turn-off time in this case is just the excited-state lifetime:

$$
\tau^{\text {off }}=2 / \Gamma
$$

Finally, for the case where the frequency of the control beam is changed without changing its intensity, we state without explicitly writing the equations that the characteristic time for the transients to decay is the same as $\tau^{\text {on }}$. The coherence also has a beat note during this transient time, oscillating at the difference frequency of the initial and final control beams.

We see that the turn-on time $\tau^{\text {on }}$ is the inverse of the Raman absorption linewidth and the turn-off time is the inverse of the homogeneous linewidth of the excited state. Hence, $\tau^{\text {off }} \ll \tau^{\text {on }} ; \tau^{\text {on }}$ is also equal to the time delay obtained for one absorption length. Thus, for a system with a delay-bandwidth product of 1 , we can change the slow-light parameters at the bit rate of the pulse stream and we can turn off the slow light within a time that is much less than the pulse width. Such fast dynamics makes this scheme an attractive candidate for pulse synchronization techniques.

\section{EXPERIMENT}

We demonstrate the dual Raman absorption slow light using warm atomic rubidium vapor. The outline of the experimental setup is depicted in Fig. 1(a). The 795-nm laser is composed of a narrow-linewidth tunable diode laser followed by a tapered amplifier to obtain a high-power laser beam. The frequency of the laser is tuned to the required frequency of the coupling beam. Probe $P$ is obtained by frequency-shifting part of the the laser beam by $3.035 \mathrm{GHz}$ by double-passing it through a tunable $1.5 \mathrm{GHz}$ acousto-optic modulator (AOM1). The other part of the laser beam is further split and sent through two $80-\mathrm{MHz}$ AOMs resulting in two coupling beams, $C 1$ and $C 2$. The frequency difference between $C 1$ and $C 2$ is controlled by changing the radio frequency fed to these AOMs. The coupling beams $C 1$ and $C 2$ are combined with the probe at the polarizing beam splitters PB2 and PB4 in front of the vapor cells VC1 and $\mathrm{VC} 2$, respectively. Beam $C 1$ is filtered from the probe at PB3 before VC2 and $C 2$ is filtered at PB5, placed after vapor cell VC2. The probe is measured at the photodetector PD.

Vapor cells VC1 and VC2 are identical 5-cm-long vapor cells placed inside hollow $\mathrm{Mu}$-metal tubes to block all the stray magnetic fields. The vapor cells are heated by strip heaters and the current passing through the strip heaters is identical for each cell. Each cell along with the Mu-metal tubing and the heaters is placed inside a teflon tube enclosed by antireflectioncoated windows at each end. The vapor cells contain both rubidium isotopes in their natural abundance. In addition, we also have 20 Torr neon in each cell, which acts as a buffer gas. The temperature of each vapor cell is about $80^{\circ} \mathrm{C}$, resulting in a number density of about $10^{12} \mathrm{~cm}^{-3}$ in each cell.

Figure 2 shows the experimental observation of the delay. The frequency difference between two coupling beams in VC1 and $\mathrm{VC} 2$ is $3 \mathrm{MHz}$. The alignment of the coupling and probe beams is adjusted for each vapor cell to obtain the Raman
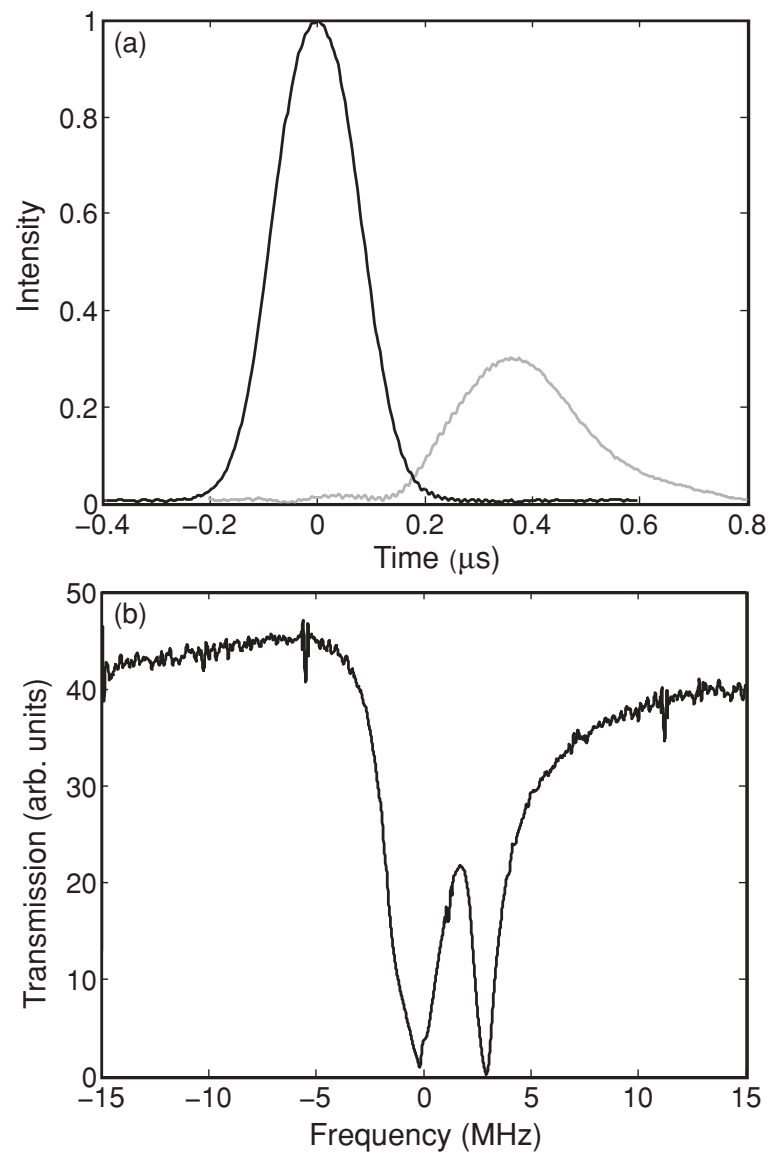

FIG. 2. (a) The black line is the reference pulse, and the gray line is the delayed pulse. (b) The transmission profile of the probe corresponding to the observed delay.

absorption dip. Since the two coupling beams are detuned, we observe that the transmission of the probe has two absorption dips, as shown in Fig. 2(b). The probe pulse with full width at half maximum (FWHM) of about $179 \mu$ s is tuned to the center of the transparency in between the absorption dips. We observe a delay of about $374 \mathrm{~ns}$ with the coupling beams on. This corresponds to a delay bandwidth product of about 2 (bandwidth here is measured as FWHM of the pulse). The FWHM of the output pulse is about 262 ns and, thus, has a broadening factor of 1.4. Thus, we demonstrate slow group velocity with relatively less pulse broadening using the two coupling-induced absorption dips.

Figure 3 shows the experimental observations of switching times. In order to measure turn-off time, the probe pulse tuned to the center of Raman absorption is sent through the medium with the coupling beam on and, as the pulse is propagating inside the medium, the coupling beam is turned off. As we see in Fig. 3(a), the pulse has lower transmission before turning off the coupling beam because of absorption due to coupling. After the coupling beam is turned of,f the probe transmission increases to match the dotted line within 50 ns and the rise time is limited only by the rise time of the AOM used to switch the coupling beam. The turn-on time is measured by having the coupling beam initially off and turning it on as the probe is inside the medium, as shown in Fig. 3(a). The turn-on time is measured to be about $0.75 \mu \mathrm{s}$. The transients for the 

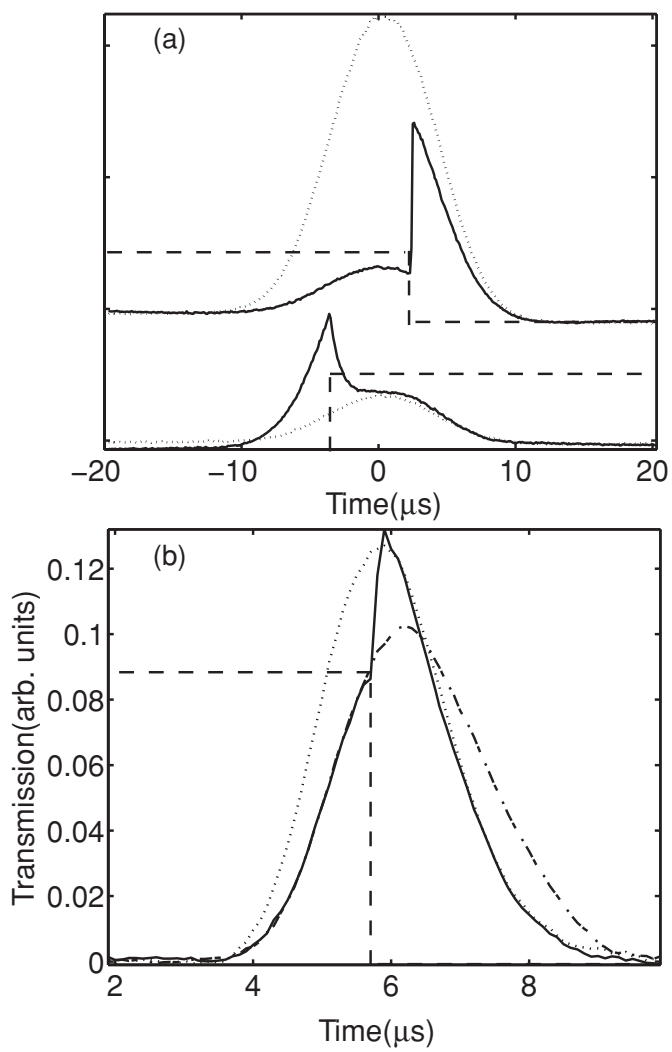

FIG. 3. (a) The dotted curve at the top shows the reference pulse and the one at the bottom shows the output pulse in the presence of coupling-induced absorption. The dashed line shows the coupling level. The solid line at the top shows the turn-off characteristics of the absorption and at the bottom the turn-on characteristics. (b) The experimental observation of slow-light switching. The dotted line is the input pulse without any delay and the broken line is the output pulse with delay. The black line demonstrates the slow-light switching. The black dashed line indicates the level of control fields. change in frequency of the coupling beam have a similar time constant and we see modulation in the signal during transition time. Figure 3(b) shows the experimental observation of slowlight switching. The dotted line is the input pulse without any delay and the broken line is the output pulse with delay. The black line demonstrates the slow-light switching. As the pulse is compressed inside the vapor cell, we turn off the control fields as shown by the black dashed line. We turn off the slow propagation of the pulse all optically within $50 \mathrm{~ns}$, demonstrating high-speed switching of slow light.

\section{CONCLUSIONS}

We have presented a double-absorption slow-light system based on the Raman absorption dip. The strength, the width, and the center of the absorption can all be modified optically by changing the properties of the coupling beam. In addition, we can even tune the number density and the single-photon detuning to further modify the properties of the slow-light system. The wide range of independent controls makes this slow-light system versatile. However, the limitation to this versatility is imposed by the competing nonlinear four-wave mixing process, which dominates at high atomic number densities. This limitation is addressed to some extent in this paper by careful alignment of the probe and coupling beams in order to have a phase matching that favors absorption and suppresses four-wave mixing. Also we avoid the problem of multiwave mixing resulting from having two coupling beams and the probe beam in one vapor cell by using two vapor cells.

\section{ACKNOWLEDGMENTS}

We gratefully acknowledge support from DARPA Expansion Grant No. N00014-08-1-120 and the University of Rochester.
[1] P. Ku, C. Chang-Hasnain, and S. Chuang, Electron. Lett. 38, 1581 (2002).

[2] R. W. Boyd and D. J. Gauthier, Slow and Fast Light, Progress in Optics Vol. 43 (Elsevier, New York, 2002).

[3] P. Milonni, Fast Light, Slow Light and Left-Handed Light (Institute of Physics, University of Reading, Berkshire, 2005).

[4] J. B. Khurgin, Adv. Opt. Photon. 2, 287 (2010).

[5] L. V. Hau, S. E. Harris, Z. Dutton, and C. H. Behroozi, Nature (London) 397, 594 (1999).

[6] M. M. Kash, V. A. Sautenkov, A. S. Zibrov, L. Hollberg, G. R. Welch, M. D. Lukin, Y. Rostovtsev, E. S. Fry, and M. O. Scully, Phys. Rev. Lett. 82, 5229 (1999).

[7] D. Budker, D. F. Kimball, S. M. Rochester, and V. V. Yashchuk, Phys. Rev. Lett. 83, 1767 (1999).

[8] R. M. Camacho, M. V. Pack, and J. C. Howell, Phys. Rev. A 73, 063812 (2006).

[9] A. V. Turukhin, V. S. Sudarshanam, M. S. Shahriar, J. A. Musser, B. S. Ham, and P. R. Hemmer, Phys. Rev. Lett. 88, 023602 (2001).
[10] M. S. Bigelow, N. N. Lepeshkin, and R. W. Boyd, Phys. Rev. Lett. 90, 113903 (2003).

[11] M. S. Bigelow, N. N. Lepeshkin, and R. W. Boyd, Science 301, 200 (2003).

[12] Y. Okawachi, M. S. Bigelow, J. E. Sharping, Z. Zhu, A. Schweinsberg, D. J. Gauthier, R. W. Boyd, and A. L. Gaeta, Phys. Rev. Lett. 94, 153902 (2005).

[13] S. Residori, U. Bortolozzo, and J. P. Huignard, Phys. Rev. Lett. 100, 203603 (2008).

[14] T. Baba, Nat Photonics 2, 465 (2008).

[15] Z. Shi, R. W. Boyd, R. M. Camacho, P. K. Vudya Setu, and J. C. Howell, Phys. Rev. Lett. 99, 240801 (2007).

[16] Z. Shi and R. W. Boyd, J. Opt. Soc. Am. B 25, C136 (2008).

[17] M. S. Shahriar, G. S. Pati, R. Tripathi, V. Gopal, M. Messall, and K. Salit, Phys. Rev. A 75, 053807 (2007).

[18] Z. Zhu and D. J. Gauthier, Opt. Express 14, 7238 (2006). 
[19] M. Fleischhauer, A. Imamoglu, and J. P. Marangos, Rev. Mod. Phys. 77, 633 (2005).

[20] E. E. Mikhailov, V. A. Sautenkov, Y. V. Rostovtsev, and G. R. Welch, J. Opt. Soc. Am. B 21, 425 (2004).

[21] S. Knappe, M. Stähler, C. Affolderbach, A. Taichenachev, V. Yudin, and R. Wynands, Appl. Phys. B 76, 57 (2003).
[22] K. F. Reim, J. Nunn, V. O. Lorenz, B. J. Sussman, K. C. Lee, N. K. Langford, D. Jaksch, and I. A. Walmsley, Nat. Photonics 4, 218 (2010).

[23] N. A. Proite, B. E. Unks, J. T. Green, and D. D. Yavuz, Phys. Rev. Lett. 101, 147401 (2008). 